\title{
THE DIRICHLET PROBLEM FOR THE TIME-FRACTIONAL ADVECTION-DIFFUSION EQUATION IN A HALF-SPACE
}

\author{
Yuriy Povstenko ${ }^{1}$, Joanna Klekot ${ }^{2}$ \\ ${ }^{I}$ Institute of Mathematics and Computer Science, Jan Dlugosz University in Czestochowa \\ Częstochowa, Poland \\ ${ }^{2}$ Institute of Mathematics, Czestochowa University of Technology \\ Częstochowa, Poland \\ j.povstenko@ajd.czest.pl,joanna.klekot@im.pcz.pl
}

\begin{abstract}
The one-dimensional time-fractional advection-diffusion equation with the Caputo time derivative is considered in a half-space. The fundamental solution to the Dirichlet problem and the solution of the problem with constant boundary condition are obtained using the integral transform technique. The numerical results are illustrated graphically.
\end{abstract}

Keywords: Caputo fractional derivative, advection-diffusion equation, Laplace integral transform, Fourier sine transform, Mittag-Leffler function

\section{Introduction}

Fractional calculus (the theory of integrals and derivatives of arbitrary order) has many applications in different areas of physics, biology and engineering [1-5]. The time-fractional advection-diffusion equation

$$
\frac{\partial^{\alpha} c(x, t)}{\partial t^{\alpha}}=a \Delta c-\mathbf{v} \cdot \operatorname{grad} c
$$

describes diffusion or heat conduction with additional velocity field, transport processes in porous media or groundwater hydrology. This equation can be obtained as a consequence of the balance equation for mass and the time-nonlocal constitutive equation for the matter flux with the "long-tail" power kernel [6] (compare the analysis of [6] with the analysis of the generalized Fourier or Fick law carried out in [7-10]). The comprehensive survey of literature on the fractional advection-diffusion equation can be found in [11]. In the previous paper [12] the fundamental solution to the Cauchy problem for time-fractional advection-diffusion equation with one spatial variable was obtained in the domain $-\infty<x<\infty$. In the present paper we study the Dirichlet problem for this equation in a half-line $0<x<\infty$. Two types of boundary conditions are considered: the Dirac delta boundary condi- 
tion for the fundamental solution and the constant boundary condition for the sought-for function.

\section{The fundamental solution to the Dirichlet problem}

We consider the time-fractional advection-diffusion equation

$$
\frac{\partial^{\alpha} c(x, t)}{\partial t^{\alpha}}=a \frac{\partial^{2} c(x, t)}{\partial x^{2}}-v \frac{\partial c(x, t)}{\partial x}
$$

where $0<\alpha \leq 1,0<x<\infty, 0<t<\infty, a>0, v>0$. In equation (2) $\partial^{\alpha} c / \partial t^{\alpha}$ is the Caputo fractional derivative of the order $\alpha$ [13]:

$$
\frac{\partial^{\alpha} c}{\partial t^{\alpha}}=\frac{1}{\Gamma(n-\alpha)} \int_{0}^{t}(t-\tau)^{n-\alpha-1} \frac{\partial^{n} c(\tau)}{\partial \tau^{n}} d \tau, \quad n-1<\alpha<n,
$$

where $\Gamma(x)$ is the gamma function. Equation (3) simplifies for $0<\alpha<1$ :

$$
\frac{\partial^{\alpha} c}{\partial t^{\alpha}}=\frac{1}{\Gamma(1-\alpha)} \int_{0}^{t} \frac{1}{(t-\tau)^{\alpha}} \frac{\partial c(\tau)}{\partial \tau} d \tau
$$

The equation (2) is considered under zero initial condition

$$
c(x, 0)=0,
$$

and the Dirichlet boundary condition

$$
c(0, t)=g_{0} \delta(t)
$$

with $\delta(t)$ being the Dirac delta function. In the above condition we have introduced the constant multiplier $g_{0}$ to obtain the nondimensional quantity displayed in figures.

The zero condition at infinity is imposed as follows:

$$
\lim _{x \rightarrow \infty} c(x, t)=0 .
$$

Introducing the new sought-for function

$$
c(x, t)=\exp \left(\frac{v x}{2 a}\right) u(x, t),
$$


the initial-boundary-value problem (2), (5)-(7) is reduced to the following one:

$$
\begin{gathered}
\frac{\partial^{\alpha} u(x, t)}{\partial t^{\alpha}}=a \frac{\partial^{2} u(x, t)}{\partial x^{2}}-\frac{v^{2}}{4 a} u(x, t), \\
u(x, 0)=0, \\
u(0, t)=g_{0} \delta(t), \\
\lim _{x \rightarrow \infty} u(x, t)=0 .
\end{gathered}
$$

To solve the Dirichlet problem under consideration we use the Fourier sine transform with respect to the spatial coordinate $x$ :

$$
\begin{gathered}
F\{u(x)\}=\tilde{u}(\xi)=\int_{0}^{\infty} u(x) \sin (\xi x) d x, \\
F^{-1}\{\tilde{u}(\xi)\}=u(x)=\frac{2}{\pi} \int_{0}^{\infty} \tilde{u}(x) \sin (\xi x) d \xi .
\end{gathered}
$$

The Fourier sine transform of the second order derivative of a function is defined by the relation:

$$
F\left\{\frac{d^{2} u(x)}{d t^{2}}\right\}=-\xi^{2} \tilde{u}(\xi)+\xi u(0)
$$

Application of the Fourier sine transform (13) to equation (9) using (15) leads to

$$
\frac{\partial^{\alpha} \tilde{u}(\xi, t)}{\partial t^{\alpha}}=\left(-a \xi^{2}-\frac{v^{2}}{4 a}\right) \tilde{u}(\xi, t)+a \xi g_{0} \delta(t) \text {. }
$$

Next, we use the Laplace transform with respect to the time $t$. For the function $u(t), 0<t<\infty$, this transform is defined by

$$
L\{u(t)\}=u^{*}(s)=\int_{0}^{\infty} e^{-s t} u(t) d t
$$


with the inverse carrying out according to the Fourier-Mellin formula

$$
L^{-1}\left\{u^{*}(s)\right\}=u(t)=\frac{1}{2 \pi i} \int_{\gamma-i \infty}^{\gamma+i \infty} e^{s t} u^{*}(s) d s,
$$

where $\gamma$ is a positive fixed number.

For the Laplace transform rule the Caputo fractional derivative requires the knowledge of the initial values of the function and its integer derivatives of the order $k=1,2, \ldots, n-1$ :

$$
L\left\{\frac{d^{\alpha} u(t)}{d t^{\alpha}}\right\}=s^{\alpha} u^{*}(s)-\sum_{k=0}^{n-1} s^{\alpha-1-k} u^{(k)}\left(0^{+}\right), \quad n-1<\alpha \leq n .
$$

Equation (19) simplifies for $0<\alpha<1$ :

$$
L\left\{\frac{d^{\alpha} u(t)}{d t^{\alpha}}\right\}=s^{\alpha} u^{*}(s)-s^{\alpha-1} u\left(0^{+}\right) .
$$

Applying the Laplace transform to equation (17) and taking into account the rule (20) with the initial condition (10) gives

$$
s^{\alpha} \tilde{u}^{*}(\xi, s)=\left(-a \xi^{2}-\frac{v^{2}}{4 a}\right) \tilde{u}^{*}(\xi, s)+a g_{0} \xi .
$$

In the transform domain we get:

$$
\tilde{u}^{*}(\xi, s)=a g_{0} \frac{\xi}{s^{\alpha}+a \xi^{2}+\frac{v^{2}}{4 a}} .
$$

Inversion of the integral transforms results in the solution:

$$
u(x, t)=\frac{2 a g_{0}}{\pi} t^{\alpha-1} \int_{0}^{\infty} E_{\alpha, \alpha}\left[-\left(a \xi^{2}+\frac{v^{2}}{4 a}\right) t^{\alpha}\right] \xi \sin (\xi x) d \xi,
$$

where the formula [8]

$$
L^{-1}\left\{\frac{s^{\alpha-\beta}}{s^{\alpha}+b}\right\}=t^{\beta-1} E_{\alpha, \beta}\left(-b t^{\alpha}\right)
$$


has been used with $E_{\alpha, \beta}(z)$ being the Mittag-Leffler function in two parameters $\alpha, \beta$ and having the series representation

$$
E_{\alpha, \beta}(z)=\sum_{n=0}^{\infty} \frac{z^{n}}{\Gamma(\alpha n+\beta)}, \quad \alpha, \beta>0
$$

Returning to the quantity $c(x, t)$ according to (8), we get

$$
c(x, t)=\frac{2 a g_{0}}{\pi} \exp \left(\frac{v x}{2 a}\right) t^{\alpha-1} \int_{0}^{\infty} E_{\alpha, \alpha}\left[-\left(a \xi^{2}+\frac{v^{2}}{4 a}\right) t^{\alpha}\right] \xi \sin (\xi x) d \xi .
$$

The quantities appearing in the fractional advection-diffusion equation (2) and the boundary condition (5) have the following physical units: $[c]=\mathrm{kg} / \mathrm{m}^{3}$, $[x]=\mathrm{m},[t]=\mathrm{s},[a]=\mathrm{m}^{2} / \mathrm{s}^{\alpha},[v]=\mathrm{m} / \mathrm{s}^{\alpha},\left[g_{0}\right]=\mathrm{kg} \cdot \mathrm{s} / \mathrm{m}^{3}$. At a level of individual particle motion the classical diffusion $(\alpha=1)$ corresponds to the Brownian motion which is characterized by a mean-squared displacement increasing linearly with time

$$
\left\langle x^{2}\right\rangle \approx a t \text { with }[a]=\mathrm{m}^{2} / \mathrm{s}
$$

Anomalous diffusion is exemplified by a mean-squared displacement with the power law time dependence

$$
\left\langle x^{2}\right\rangle \approx a t^{\alpha} \text { with }[a]=\mathrm{m}^{2} / \mathrm{s}^{\alpha}
$$

Using the nondimensional quantities

$$
\bar{x}=\frac{x}{\sqrt{a} t^{\alpha / 2}}, \quad \bar{\xi}=\sqrt{a} t^{\alpha / 2} \xi, \quad \bar{v}=\frac{v t^{\alpha / 2}}{\sqrt{a}}, \quad \bar{c}=\frac{t c}{g_{0}},
$$

one obtains the following solution:

$$
\bar{c}(\bar{x})=\frac{2}{\pi} \exp \left(\frac{\bar{v} \bar{x}}{2}\right) \int_{0}^{\infty} E_{\alpha, \alpha}\left(-\bar{\xi}^{2}-\frac{\bar{v}^{2}}{4}\right) \bar{\xi} \sin (\bar{\xi} \bar{x}) d \bar{\xi} .
$$

The results of numerical calculations for different values of the drift parameter $\bar{v}$ and the order of the time-fractional derivative $\alpha$ are shown in Figures 1-4. 


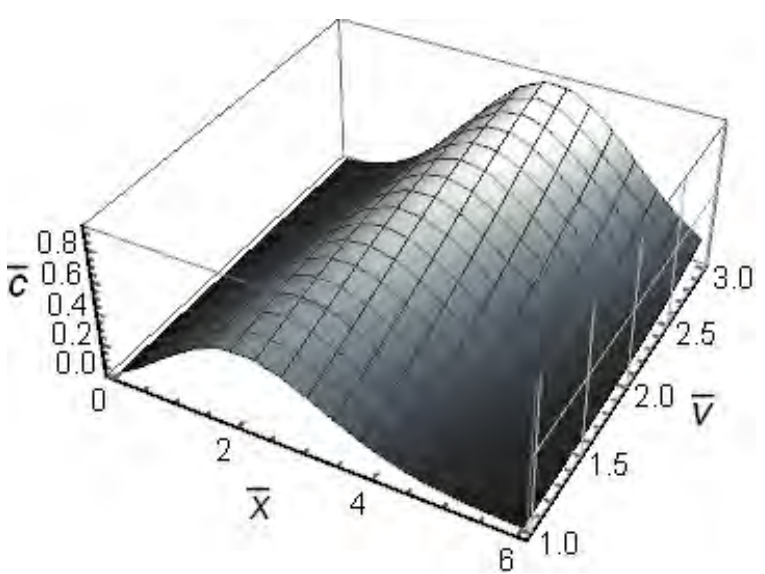

Fig. 1. The fundamental solution to the Dirichlet problem for $\alpha=1$

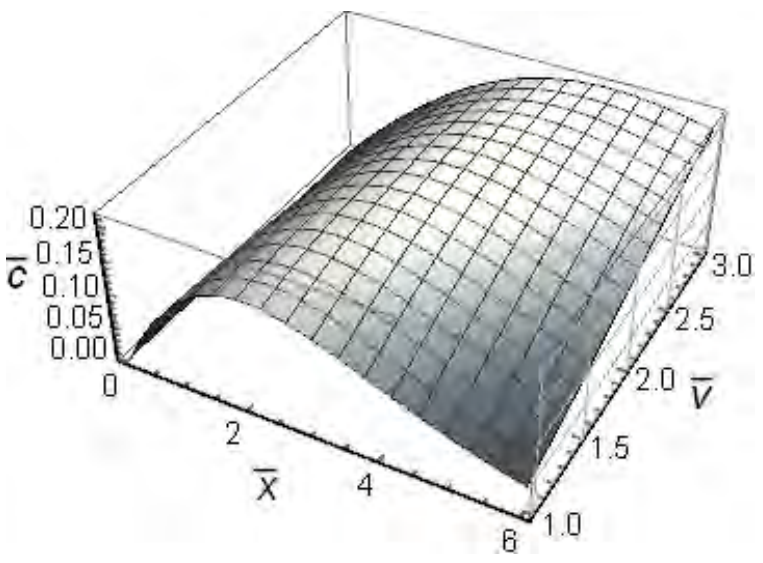

Fig. 2. The fundamental solution to the Dirichlet problem for $\alpha=0.5$

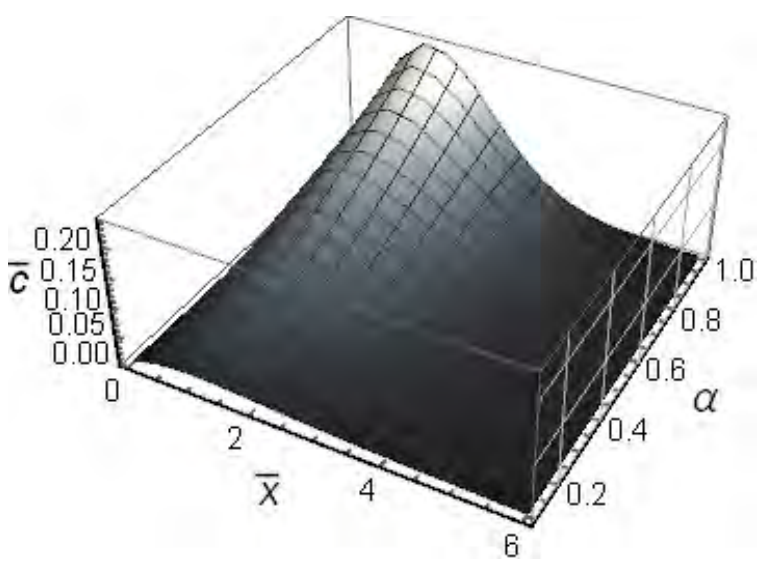

Fig. 3. The fundamental solution to the Dirichlet problem for $\bar{v}=0$ 


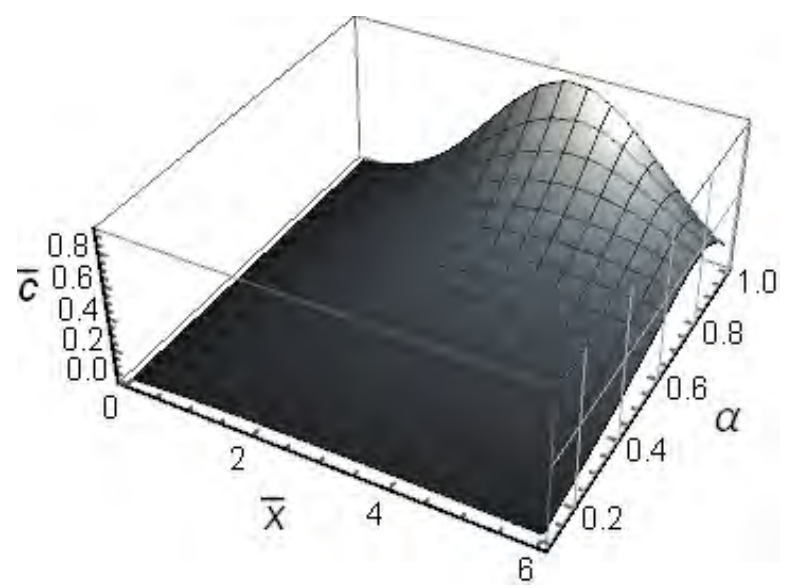

Fig. 4. The fundamental solution to the Dirichlet problem for $\bar{v}=3$

\section{Constant boundary value of a function}

Next we consider the time-fractional advection-diffusion equation (2) under zero initial condition (5), the condition (7) at infinity and the Dirichlet boundary condition with constant boundary value of the sought-for function:

$$
c(0, t)=c_{0} .
$$

As above, the new sought-for function $u$ is introduced (see (8)), and the Laplace transform with respect to time and the Fourier sine transform with respect to the spatial coordinate give the solution in the transform domain:

$$
\tilde{u}^{*}(\xi, s)=a c_{0} \frac{\xi}{s\left(s^{\alpha}+a \xi^{2}+\frac{v^{2}}{4 a}\right)} .
$$

Taking into account that

$$
\frac{1}{s\left(s^{\alpha}+a \xi^{2}+\frac{v^{2}}{4 a}\right)}=\frac{1}{a \xi^{2}+\frac{v^{2}}{4 a}}\left[\frac{1}{s}-\frac{s^{\alpha-1}}{s^{\alpha}+a \xi^{2}+\frac{v^{2}}{4 a}}\right]
$$

we obtain 


$$
\tilde{u}^{*}(\xi, s)=c_{0} \frac{\xi}{\xi^{2}+\frac{v^{2}}{4 a^{2}}}\left[\frac{1}{s}-\frac{s^{\alpha-1}}{s^{\alpha}+a \xi^{2}+\frac{v^{2}}{4 a}}\right]
$$

or, after inversion of the integral transforms,

$$
u(x, t)=\frac{2 c_{0}}{\pi} \int_{0}^{\infty} \frac{\xi \sin (\xi x)}{\xi^{2}+\frac{v^{2}}{4 a^{2}}}\left\{1-E_{\alpha}\left[-\left(a \xi^{2}+\frac{v^{2}}{4 a}\right) t^{\alpha}\right]\right\} d \xi
$$

In this case $E_{\alpha}(z) \equiv E_{\alpha, 1}(z)$ is the Mittag-Leffler function in one parameter $\alpha$ (see the series representation (25) with $\beta=1$ ).

Taking into account the following integral [14]:

$$
\int_{0}^{\infty} \frac{\xi \sin (\xi x)}{\xi^{2}+\frac{v^{2}}{4 a^{2}}} d \xi=\frac{\pi}{2} \exp \left(-\frac{v x}{2 a}\right)
$$

and returning to the quantity $c(x, t)$ according to (8), we get

$$
c(x, t)=c_{0}\left\{1-\frac{2}{\pi} \exp \left(\frac{v x}{2 a}\right) \int_{0}^{\infty} \frac{\xi \sin (\xi x)}{\xi^{2}+\frac{v^{2}}{4 a^{2}}} E_{\alpha}\left[-\left(a \xi^{2}+\frac{v^{2}}{4 a}\right) t^{\alpha}\right] d \xi\right\}
$$

and

$$
\bar{c}(\bar{x})=1-\frac{2}{\pi} \exp \left(\frac{\bar{v} \bar{x}}{2}\right) \int_{0}^{\infty} \frac{\bar{\xi} \sin (\bar{\xi} \bar{x})}{\bar{\xi}^{2}+\frac{\bar{v}^{2}}{4}} E_{\alpha}\left(-\bar{\xi}^{2}-\frac{\bar{v}^{2}}{4}\right) d \bar{\xi}
$$

where

$$
\bar{c}=\frac{c}{c_{0}}
$$

other nondimensional quantities are the same as in (27).

The results of numerical calculations according to the solution (36) are shown in Figures 5-8 for different values of the drift parameter $\bar{v}$ and the order of the time-fractional derivative $\alpha$. 


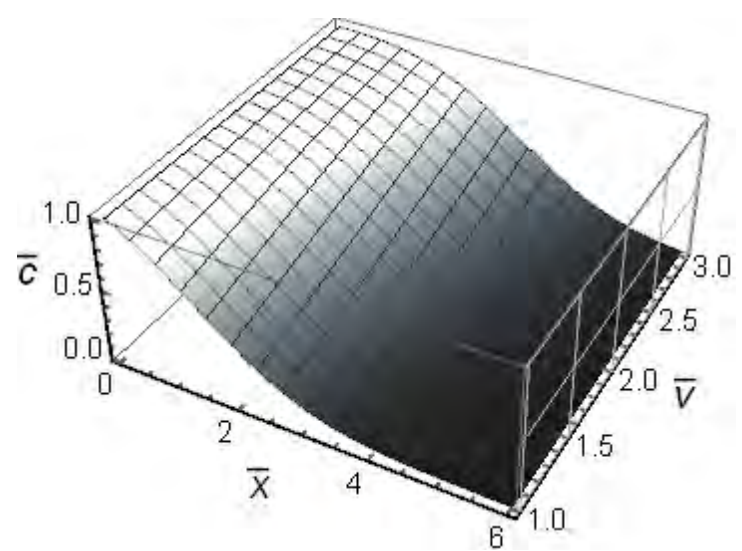

Fig. 5. The solution to the Dirichlet problem with constant boundary value of a function for $\alpha=1$

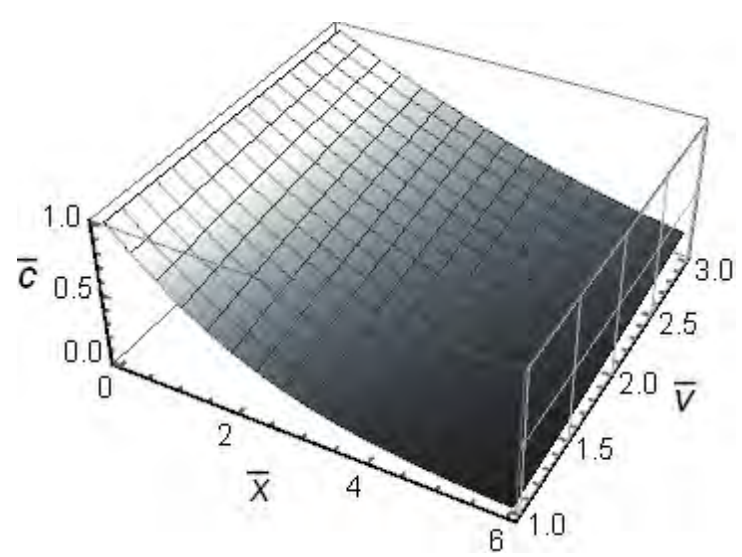

Fig. 6. The solution to the Dirichlet problem with constant boundary value of a function for $\alpha=0.5$

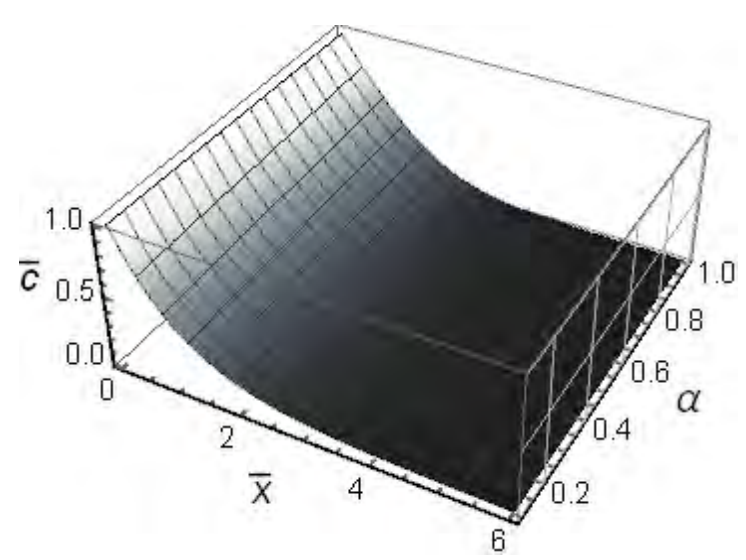

Fig. 7. The solution to the Dirichlet problem with constant boundary value of a function for $\bar{v}=0$ 


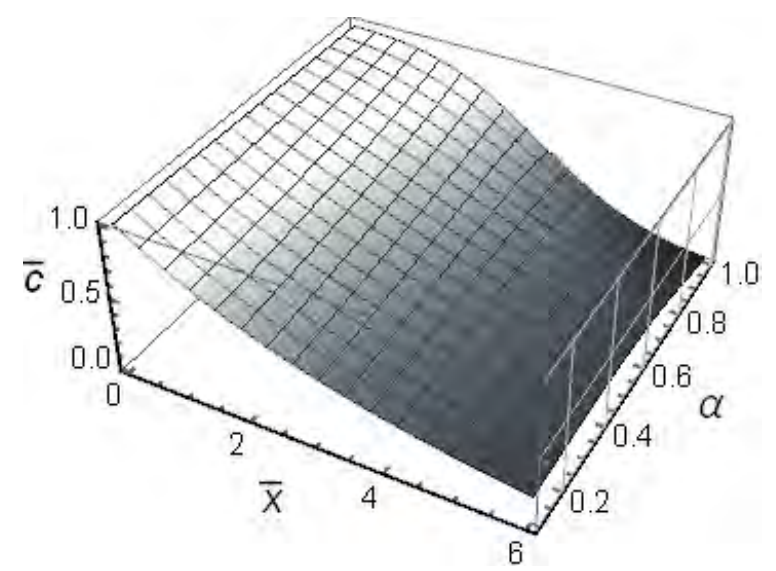

Fig. 8. The solution to the Dirichlet problem with constant boundary value of a function for $\bar{v}=3$

\section{Conclusions}

We have considered the time-fractional advection-diffusion equation with the Caputo fractional derivative in the case of one spatial coordinate in the semi-infinite domain $0<x<\infty$. The Laplace transform with respect to time and the Fourier sine transform with respect to the spatial coordinate have been used. The fundamental solution to the Dirichlet problem and the solution to the problem with constant boundary condition for the sought-for function have been obtained. The results of numerical calculations are displayed in figures for different values of the drift parameter $\bar{v}$ and the order $\alpha$ of the Caputo fractional derivative. It is seen from the figures that decreasing of the order of the fractional derivative $\alpha$ (taking memory into account) leads to retardation of the mass transport process (both the pure diffusion and the influence of the drift parameter). This is due to the complicated internal structure of the modeled medium (the presence of pores, inclusions, combs, etc.) which causes the memory effects. The influence is more noticeable for the fundamental solution than in the case of constant boundary condition. The numerical calculations were carried out using the package Mathematica.

\section{References}

[1] Hilfer R. (ed.), Applications of Fractional Calculus in Physics, World Scientific, Singapore 2000.

[2] West B.J., Bologna M., Grigolini P., Physics of Fractals Operators, Springer, New York 2003.

[3] Magin R.L., Fractional Calculus in Bioengineering, Begell House Publishers, Connecticut 2006.

[4] Ghazizadeh H.R., Maerefat M., Modeling diffusion to thermal wave heat propagation by using fractional heat conduction constitutive model, Iranian Journal of Mechanical Engineering 2010, 11(2), 66-80.

[5] Uchaikin V.V., Fractional Derivatives for Physicists and Engineers, Springer, Berlin 2013. 
[6] Povstenko Y., Theory of diffusive stresses based on the fractional advection-diffusion equation, [in:] Fractional Calculus: Applications, eds. R. Abi Zeid Daou, X. Moreau, NOVA Science Publisher, New York 2015, 227-241.

[7] Povstenko Y., Fractional heat conduction equation and associated thermal stresses, J. Thermal Stresses 2005, 28, 83-102.

[8] Povstenko Y., Thermoelasticity which uses fractional heat conduction equation, J. Math. Sci. 2009, 162, 296-305.

[9] Povstenko Y., Theory of thermoelasticity based on the space-time-fractional heat conduction equation, Phys. Scr. T 2009, 136, 014017.

[10] Povstenko Y., Fractional thermoelasticity, [in:] Encyclopedia of Thermal Stresses, ed. R.B. Hetnarski, Springer, New York 2014, 1778-1787.

[11] Povstenko Y., Fundamental solutions to time-fractional advection diffusion equation in a case of two space variables, Math. Probl. Eng. 2014, 2014, 705364.

[12] Povstenko Y., Klekot J., Fundamental solution to the Cauchy problem for the time-fractional advection-diffusion equation, J. Appl. Math. Comput. Mech. 2014, 13, 95-102.

[13] Kilbas A., Srivastava H., Trujillo J., Theory and Applications of Fractional Differential Equations, Elsevier, Amsterdam 2006.

[14] Prudnikov A.P., Brychkov Yu.A., Marichev O.I., Integrals and Series, Vol. 1. Elementary Functions, Gordon and Breach, Amsterdam 1986. 